\title{
An Extraordinary Case of Silent Extensive Anterior Wall Myocardial Infarction Complicated with Giant Left Ventricular Aneurysm and Dressler Syndrome
}

\author{
Iat-Lon Leong1, Weng-Chio Tam², Paul Chan (Chen) ${ }^{2,3 *}$,Zhong-Min Liu ${ }^{3^{*}}$ \\ ${ }^{1}$ Department of Cardiology, Kan Wu Hospital, Macau, China \\ ${ }^{2}$ Division of Cardiovascular Medicine, Department of Internal Medicine, Wan Fang Hospital, Taipei Medical \\ University, Taipei, Taiwan \\ ${ }^{3}$ Department of Cardiac Surgery, Shanghai East Hospital, Tongji University, Shanghai, China \\ Email: ${ }^{*}$ chanpaul@w.tmu.edu.tw, ${ }^{*}$ Zhongmin liu@sina.com.cn
}

Received 3 April 2014; revised 6 May 2014; accepted 14 May 2014

Copyright (C) 2014 by authors and Scientific Research Publishing Inc.

This work is licensed under the Creative Commons Attribution International License (CC BY). http://creativecommons.org/licenses/by/4.0/

(c) (i) Open Access

\section{Abstract}

Early post-acute myocardial infarction (AMI) pericarditis, pericardial effusion with or without cardiac tamponade, and late post-MI pericarditis (Dressler syndrome), are the major pericardial complications after AMI. It is quite rare and estimated to be only about $0.1 \%$ in AMI patients according to a recent report, so it is easily neglected or misdiagnosed and may have tragic result to patient. Clinical features of this post-AMI complication include fever, chest pain, pericarditis and pleurisy occurring 2 to 3 weeks after AMI. Dressler syndrome is rarely associated with left ventricular aneurysm. Contrast enhanced magnetic resonance and echocardiography play important roles in diagnosis of left ventricle aneurysm. We report a 54-year-old male heavy labor worker who had asymptomatic, severe coronary artery disease, complicated with silent myocardial infarction, which resulted in large left ventricular aneurysm, and also systolic heart failure was noted. Patient was diagnosed to have Dressler syndrome after his second cardiology clinic follow-up. He received coronary angiography which revealed triple vessel disease with total occlusion of left anterior descending artery, and a giant left ventricular aneurysm was found. He received surgical intervention with Batista method and followed-up uneventfully at the cardiology clinic.

\section{Keywords}

Dressler Syndrome, Left Ventricular Aneurysm, Silent Myocardial Infarction

\footnotetext{
${ }^{*}$ Corresponding authors.
} 


\section{Introduction}

Silent myocardial infarction (MI) is not a rare entity in patients with coronary artery disease (CAD). However, silent extensive anterior wall MI complicated with giant left ventricular aneurysm (LVA) was quite rare; and this large area silent MI further complicated with Dressler syndrome was even rarer, and hitherto unreported in the previous literatures.

Dressler syndrome is the early post-acute myocardial infarction (AMI) pericarditis, pericardial effusion with or without cardiac tamponade, and late post-MI pericarditis are the major pericardial complications after AMI. It is a rare entity, which was estimated to be about $0.1 \%$ in AMI patients according to a recent report [1]. Landmark studies such as the Gruppo Italiano per lo Studio Della Sopravvivenza nell' Infarto Miocardico and Thrombolysis and Angioplasty in Myocardial Infarction study groups showed that early pericarditis after AMI occurred in approximately $5 \%$ to $7 \%$ of patients treated with thrombolytics [2] [3].

However, Dressler syndrome after AMI appears to have largely disappeared in the reperfusion era and has been reported in the only $0.5 \%$ of patients after thrombolysis [4]. The time and efficacy of reperfusion appeared to be critical factors in the pathogenesis of post-infarction pericarditis in the thrombolytic era; primary percutaneous coronary intervention (PCI) had also reduced this complication.

Under this circumstance, junior cardiologist may lack clinical experience in the diagnosis and management of this entity. We report an extraordinary case of a large silent anterior wall MI complicated with giant LVA, and subsequently resulted in Dressler syndrome but misdiagnosed at the first cardiology consultation.

\section{Case Report}

A 54-year-old male labor worker visited the emergency department of a local hospital on August 3, 2009, owing to intermittent fever for 2 weeks. He was rather healthy and did not receive any health check before. The associating symptoms were mild chest tightness, chills, and cold sweating. Antibiotics were used initially in the suspicion of sepsis. Upon physical examination, a grade II/VI systolic murmur was noted at second left sternal border, friction rub at apex, and a constant splitting of second heart sound was also noted. Electrocardiogram (ECG) showed sinus rhythm with ST elevation at precordial leads (V2 - V6). A recent myocardial infarction was suspected, though the patient did not have any chest pain, nor cold sweating, nor dyspnea at recent months. The patient was an air-conditioned mechanist and he could lift air-conditioners walking upstairs. Chest X-ray revealed marked cardiomegaly with mild blunting of bilateral costophrenic angle. Laboratory data revealed elevated C-reactive protein $9.92 \mathrm{mg} / \mathrm{dL}(0.000$ - 0.500) and erythrocyte sedimentary rate $93 \mathrm{~mm} / 1 \mathrm{hr}(0$ - 10); liver function tests, lactic dehydrogenase, albumin were normal; mild macrocytic hypochromic anemia (hemoglobin $11.3 \mathrm{~g} / \mathrm{dL}$, hematocrit 34.1\%, mean cell volume $95.1 \mathrm{fl}$ ), leukocytosis with neutrophil predominant (white blood cell 11270 /uL, neutrophil 80.4\%) were noted, 2 sets blood culture yielded negative results (Table 1). Cardiologist was consulted and the first transthoracic echocardiography revealed left ventricular ejection fraction (LVEF) was 73\%, the wall motion was normal, and mild pericardial effusion; LVA was neglected at that time. Thallium-201 myocardial perfusion scan showed severe ischemia or infarction in the apex and adjacent area, apical anterior wall and apical inferior wall. After 5 days antibiotics treatment, he was discharged with suggestion to follow-up again at cardiology clinic. He was prescribed oral medications such as candesartan, nicorandil, aspirin, and also oral antibiotics since he still had mild fever.

He was referred to our cardiology clinic on August 12, 2009 because of progressive exertional dyspnea that made him cannot work. On the second cardiology visit, mild lower limbs edema was also noted. In reviewing the previous medical history and echocardiogram, silent MI complicated with LVA was noticed. This patient was a non-smoker and had no history of hypertension and diabetes. The only risk factor for his CAD was hyperlipidemia with cholesterol $267 \mathrm{mg} / \mathrm{dL}$, high-density lipoprotein cholesterol $69 \mathrm{mg} / \mathrm{dL}$ and low-density lipoprotein cholesterol $175 \mathrm{mg} / \mathrm{dL}$ (Table 1). Another transthoracic echocardiography was performed and revealed an apical aneurysm with 68\% LVEF. Repeated ECG revealed sinus rhythm with left atrial enlargement, ST elevation in lead V2 - V5, T inversion in lead V4 - V6. He was then admitted for invasive study; coronary angiography via right femoral artery revealed CAD (triple vessel disease, total occlusion of the mid-portion of the left anterior descending (LAD) artery and right coronary artery middle portion) (Figure 1). A huge LVA was noted during systolic phase by the image of cardiac left ventriculography which involved injecting contrast media into left ventricle (Figure 2(a)). Contrast enhanced magnetic resonance angiogram (MRI) also revealed a large LVA $(9.9 \times 8.0 \mathrm{~cm}$ in coronary view) with dyskinetic wall movement, and pericardial effusion (Figure 2(b)). 
Table 1. Summary of laboratory results.

\begin{tabular}{cc}
\hline Laboratory data & value \\
\hline C-reactive protein (mg/dL) & 9.92 \\
Erythrocyte sedimentary rate (mm/1hr) & 93 \\
Alanine aminotransferase (U/L) & 25 \\
Lactic dehydrogenase (U/L) & 120 \\
Albumin (g/dL) & 4.0 \\
Hemoglobin (g/dL) & 11.3 \\
Hematocrit (\%) & 34.1 \\
White blood cell (/uL) & 11270 \\
Neutrophil segment (\%) & 80.4 \\
Total cholesterol (mg/dL) & 267 \\
high-density lipoprotein cholesterol (mg/dL) & 69 \\
low-density lipoprotein cholesterol (mg/dL) & 175 \\
\hline
\end{tabular}

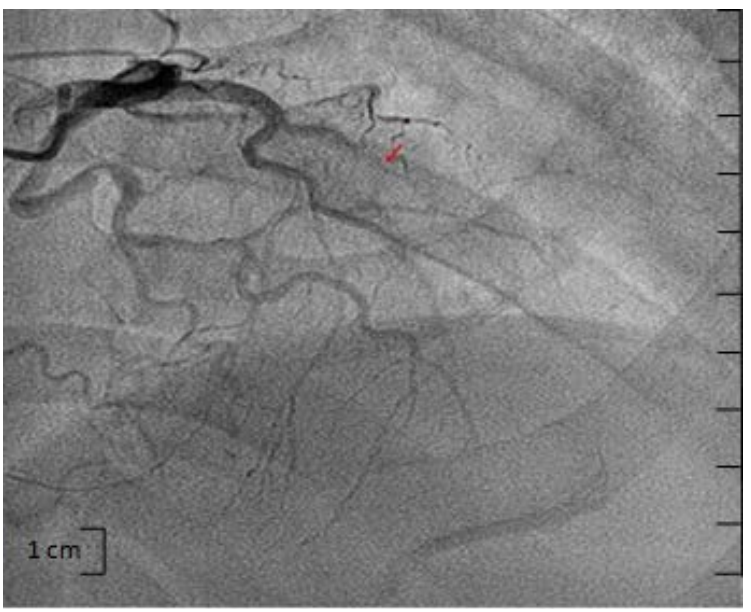

Figure 1. Coronary angiography showed total occlusion (arrow) of left anterior descending artery.

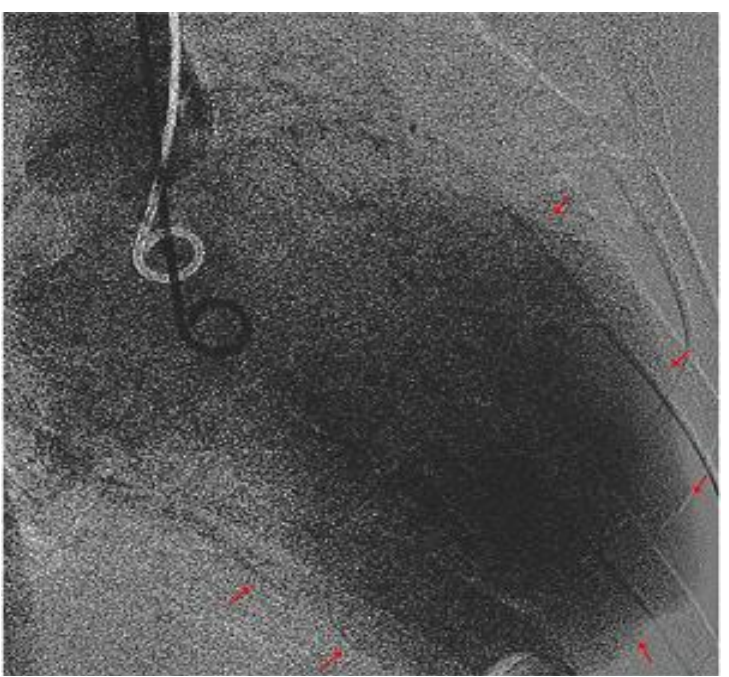

(a)

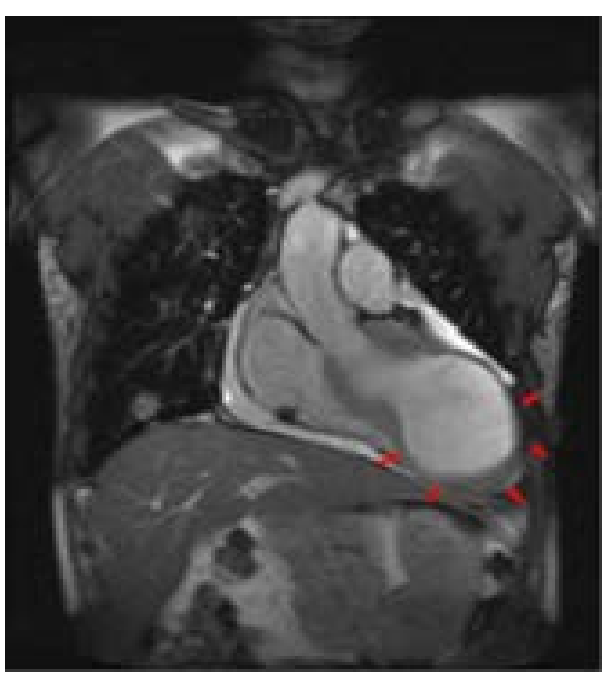

(b)

Figure 2. (a) Left ventricular angiogram showed a large aneurysm (arrows) with stagnation and whirling of blood inside the aneurysm. (b) Magnetic resonance angiography showed a large true aneurysm (arrows) at systolic phase. 
Repeated Thallium-201 myocardial perfusion scan on September 24, 2009 revealed severe ischemia or infarction in apex and adjacent area, apical anterior wall, and apical inferior wall, moderate ischemia in apical lateral wall, mild ischemia in basal lateral wall, and in comparison with prior image, deterioration of perfusion in left circumflex territory was noted. Considering about his critical condition, surgical intervention was suggested.

Surgery was performed on September 25, 2009 including coronary artery bypass grafting, left ventricular true aneurysm resection by Batista method, tricuspid annuloplasty (due to tricuspid regurgitation), and LAD artery endarectomy. Pathology report revealed ventricular wall with scar formation and granulation. Focal hemosiderin deposition with chronic inflammatory cell aggregation and mesothelial cell hyperplasia in epicardium and pericardium were also noted. After the successful surgical intervention, the patient was discharged and has been following-up at the cardiology clinic of this hospital uneventfully. He was tolerated well after surgery and mild chest discomfort without symptoms of heart failure was noted intermittently during 3 years' follow-up.

\section{Discussion}

AMI can result in the development of a dyskinetic or akinetic LVA, which may in turn cause congestive heart failure, ventricular arrhythmias and the formation of mural thrombi. True LVA occurs in approximately $7.6 \%$ of patients with CAD, most commonly as a complication of MI, in association with LAD occlusion and a wide infarcted area [5]. This patient also had total occlusion of the LAD, but this case was very unusual that he was entirely esymptomatic when his MI occurred. According to patient, he even lifted the air-conditioner by walking upstairs at about 2 weeks before his first admission. That was the Dressler syndrome which made him to be admitted to the first hospital. The reason that forced him to seek medical consultation at the second hospital was his family doctor's advice and also due to his progressive exertional dyspnea and lower leg edema.

Post-AMI syndrome was first described by Dressler in 1956 [6]. Clinical features of this post-AMI syndrome include fever, chest pain, pericarditis and pleurisy occurring 2 to 3 weeks after AMI. Since its incidence is decreasing dramatically at the reperfusion era [2]-[4]. It could be neglected or misdiagnosed easily, especially in patient with silent MI, just the same as this case we reported. This case reports a trio of AMI syndrome, namely, extensive silent MI, giant LVA and typical Dressler syndrome, which to the best of our knowledge, have not been reported elsewhere before.

Cardiac MRI has emerged as a promising noninvasive method to differentiate LV pseudoaneurysm versus true aneurysm, to localize the aneurysm, and to distinguish among pericardium, thrombus and myocardium in the aneurysm wall [7]. MRI of this patient confirmed his LV had a giant true aneurysm (Figure 2(b)). Silent MI is not an uncommon entity; patients with diabetes and heart failure are predictors of silent MI [8]. However, this patient was rather healthy before his first admission and could perform heavy labor work. In conclusion, this manuscript reports a very rare case of large area silent anterior MI complicated with LVA and Dressler Syndrome, which is unreported before.

\section{Authors' Contribution}

The authors Weng-Chio Tam contributed equally to this paper. The corresponding authors Paul Chan and Zhong-Min Liu contributed equally to this paper.

\section{References}

[1] Imazio, M., Negro, A., Belli, R., Beqaraj, F., Forno, D., Giammaria, M., Trinchero, R., Adler, Y. and Spodick, D. (2009) Frequency and Prognostic Significance of Pericarditis Following Acute Myocardial Infarction Treated by Primary Percutaneous Coronary Intervention. American Journal of Cardiology, 103, 1525-1529. http://dx.doi.org/10.1016/j.amjcard.2009.01.366

[2] Wall, T.C., Califf, R.M., Harrelson-Woodlief, L., Mark, D.B., Honan, M., Abbotsmith, C.W., et al. (1990) Usefulness of a Pericardial Friction Rub after Thrombolytic Therapy during Acute Myocardial Infarction in Predicting Amount of Myocardial Damage. American Journal of Cardiology, 66, 1418-1421. http://dx.doi.org/10.1016/0002-9149(90)90526-7

[3] Correale, E., Maggioni, A.P., Romano, S., Ricciardiello, V., Batista, R., Salvarola, G., et al. (1993) Comparison of Frequency, Diagnostic and Prognostic Significance of Pericardial Involvement in Acute Myocardial Infarction Treated with and without Thrombolytics. American Journal of Cardiology, 71, 1377-1381.

http://dx.doi.org/10.1016/0002-9149(93)90596-5 
[4] Shahar, A., Hod, H., Barabash, G.M., Kaplinsky, E. and Motro, M. (1994) Disappearance of a Syndrome: Dressler's Syndrome in the Era of Thrombolysis. Cardiology, 85, 255-258. http://dx.doi.org/10.1159/000176683

[5] Pasotti, M., Prati, F. and Arbustini, E. (2006) The Pathology of Myocardial Infarction in the Pre- and Post-Interventional Era. Heart, 92, 1552-1556. http://dx.doi.org/10.1136/hrt.2005.086934

[6] Dressler, W.A. (1956) Post-Myocardial Infarction Syndrome: Preliminary Report of a Complication Resembling Idiopathic, Recurrent, Benign Pericarditis. The Journal of the American Medical Association, 160, 1379-1383. http://dx.doi.org/10.1001/jama.1956.02960510005002

[7] Cho, M.N., Mehta, S.K., Matulevicius, S., Weinstein, D., Wait, M.A. and McGuire, D.K. (2006) Differentiating True Versus Pseudo Left Ventricular Aneurysm: A Case Report and Review of Diagnostic Strategies. Cardiology in Review, 14, e27-e30.

[8] Feringa, H.H., Karagiannis, S.E., Vidakovic, R., Elhendy, A., Folkert, J., Noordzij, P.G., et al. (2007) The Prevalence and Prognosis of Unrecognized Myocardial Infarction and Silent Myocardial Ischemia in Patients Undergoing Major Vascular Surgery. Coronary Artery Disease, 18, 571-576. http://dx.doi.org/10.1097/MCA.0b013e3282f08e86 